\title{
Allometric Assessment of Some Morphological Characters of Rainbow Trout (Oncorhynchus mykiss) Reared in Different Farming Systems from Transylvania, Romania
}

\author{
Daniel COCAN*, Vioara MIREŞAN, Radu CONSTANTINESCU, Camelia RĂDUCU, Octavian NEGREA, \\ Luisa ANDRONIE, Andrada IHUȚ
}

Faculty of Animal Science and Biotechnologies, University of Agricultural Sciences and Veterinary Medicine, 3-5 Mănăştur Street, 400372 Cluj-Napoca, Romania

*Corresponding author, email: cocandaniel@yaho.com

Bulletin UASVM Animal Science and Biotechnologies 71(2) / 2014,

Print ISSN 1843-5262; Electronic ISSN 1843-536X

DOI: $10.15835 /$ buasvmcn-asb:10788

\begin{abstract}
Whereas the growth dynamics of fish in recirculating systems is superior to conventional systems, we performed based on allometric scaling techniques, an estimate of future fisheries production in both systems. Based on the somatic measurements and body size indices, were calculated correlations ( $\mathrm{r}$ ), the regression lines (y) and determination coefficients $\left(\mathrm{R}^{2}\right)$. All the values obtained, are favorable to production obtained in a recirculating system (experimental group - E).
\end{abstract}

Keywords: allometry, rainbow trout, farming systems

Introduction. Fish morphometry is used since the Middle Ages, but if it was used only as a basic research, today presents a highly practical character.

Allometric relationship reflects the change ratios (or relative organ weight) during ontogenetic and phylogenetic development. This science is commonly used in fisheries area to determine the future fish production (Post and Parkinson, 2001; Howland et al., 2004).

Randall (2002), considers that the ratio of annual production rate to average biomass $(\mathrm{P} / \mathrm{B})$ is sometimes used as a shortcut method of estimating production if biomass is known. $\mathrm{P} / \mathrm{B}$ ratios vary among salmonid populations in different areas depending on the species, fish size and growing conditions.

Aims and objectives. The aim of this study was to determine the future fishery production based on allometric relationships, according to the system growth of rainbow trout (Oncorhynchus mykiss). Our objectives were to determine the links between body weight of trout and different phenotypic characters, by calculating correlations, regression lines and coefficients of determination.

Materials and methods. Were taken into studied two experimental groups, each of them containing 600 specimens of rainbow trout (Oncorhynchus mykiss). First group (C-control group) has been reared in classical trout farm from Fiad, Bistriţa-Năsăud County. The second group (E-experimental group) was reared in a superintensive recirculating system in ClujNapoca, Cluj County. Both groups have common origin and benefited from the same feed structure.

Results and Discussion. Due to optimal environmental conditions from recirculating system, the specimens from group E have superior growth dynamic, compared to group C. Because of this, have resulted superior values for group $\mathrm{E}$, in terms of body size indices. Using allometric techniques, we determined correlations (r), the 
Tab. 1. Correlations, regression lines and determination coefficients obtained between body weight and different phenotypic characters of rainbow trout rearing in different farming systems (2012)

\begin{tabular}{ccccccc}
\hline \multicolumn{7}{c}{ Allometric data -2012} \\
\hline Specification & $\begin{array}{c}\mathrm{r} \\
\text { C group }\end{array}$ & $\begin{array}{c}\mathrm{r} \\
\text { E group }\end{array}$ & $\begin{array}{c}\mathrm{y} \\
\text { C group }\end{array}$ & $\begin{array}{c}\mathrm{y} \\
\text { E group }\end{array}$ & $\begin{array}{c}\mathrm{R}^{2} \\
\text { C group }\end{array}$ & $\begin{array}{c}\mathrm{R}^{2} \\
\text { E group }\end{array}$ \\
\hline Bw-Sl & 0.978 & 0.975 & $0.0775 \mathrm{x}+10.332$ & $0.0451 \mathrm{x}+12.629$ & 0.9570 & 0.9507 \\
\hline Bw-Bd & 0.996 & 0.950 & $0.0187 \mathrm{x}+0.9478$ & $0.0089 \mathrm{x}+1.6886$ & 0.9921 & 0.9041 \\
\hline Bw-H & 0.990 & 0.953 & $0.0303 \mathrm{x}+2.1249$ & $0.0134 \mathrm{x}+3.4178$ & 0.9818 & 0.9095 \\
\hline Bw-P & 0.994 & 0.960 & $0.0887 \mathrm{x}+5.7393$ & $0.0397 \mathrm{x}+9.0394$ & 0.9888 & 0.9220 \\
\hline Bw-Hl & 0.953 & 0.990 & $0.0119 \mathrm{x}+2.5015$ & $0.0092 \mathrm{x}+2.7158$ & 0.9097 & 0.9820 \\
\hline Bw-Cpl & 0.775 & 0.980 & $0.0084 \mathrm{x}+2.1830$ & $0.0067 \mathrm{x}+2.2208$ & 0.6014 & 0.9622 \\
\hline
\end{tabular}

Note: Bw - body weight; Sl - standard length; Bd - Body depth; H - maximum height; P - great perimeter; Hl - head length; Cpl - caudal peduncle length; $r$ - correlations; $y$ - regression lines; $\mathrm{R}^{2}$ - coefficients of determination; $\mathrm{C}$ - control group; $\mathrm{E}$ - experimental group.

Tab. 2. Correlations, regression lines and determination coefficients obtained between body weight and different phenotypic characters of rainbow trout rearing in different farming systems (2013)

\begin{tabular}{|c|c|c|c|c|c|c|}
\hline \multicolumn{7}{|c|}{ Allometric data - 2013} \\
\hline & $r$ & $r$ & $\mathrm{y}$ & $\mathrm{y}$ & $\overline{\mathrm{R}^{2}}$ & $\overline{\mathrm{R}^{2}}$ \\
\hline Specification & C group & E group & $\mathrm{C}$ group & E group & C group & E group \\
\hline Bw-Sl & 0.973 & 0.972 & $0.0703 x+10.756$ & $0.0445 x+12.633$ & 0.9477 & 0.9453 \\
\hline $\mathrm{Bw}-\mathrm{Bd}$ & 0.987 & 0.956 & $0.0165 x+1.0606$ & $0.0087 x+1.7099$ & 0.9743 & 0.9147 \\
\hline $\mathrm{Bw}-\mathrm{H}$ & 0.978 & 0.952 & $0.0266 x+2.3383$ & $0.0131 x+3.446$ & 0.9573 & 0.9081 \\
\hline $\mathrm{Bw}-\mathrm{P}$ & 0.981 & 0.960 & $0.0769 x+6.3621$ & $0.0388 x+9.1217$ & 0.9624 & 0.9216 \\
\hline $\mathrm{Bw}-\mathrm{Hl}$ & 0.954 & 0.989 & $0.0115 x+2.5461$ & $0.0091 x+2.7059$ & 0.9106 & 0.9797 \\
\hline Bw-Cpl & 0.8261 & 0.9759 & $0.0078 x+2.2252$ & $0.0067 x+2.1792$ & 0.6825 & 0.9525 \\
\hline
\end{tabular}

regression lines (y) and determination coefficients $\left(\mathrm{R}^{2}\right)$ (Tab. 1, 2), between body weight (Bw) and body standard length $(\mathrm{Sl})$ : group $\mathrm{C}(\mathrm{r}=0.9780$; $\left.\mathrm{y}=0.0775 \mathrm{x}+10.332 ; \quad \mathrm{R}^{2}=0.9570\right)$, vs. group $\mathrm{E}$ $\left(\mathrm{r}=0.9570 ; \quad \mathrm{y}=0.0451 \mathrm{x}+12.629 ; \quad \mathrm{R}^{2}=0.9507\right) ;$ body weight (Bw) and body depth (Bd): group C $\quad\left(r=0.9960 ; \quad y=0.0187 x+0.9478 ; \quad R^{2}=0.9921\right)$, vs. group E ( $\mathrm{r}=0.9500 ; \mathrm{y}=0.0089 \mathrm{x}+1.6886$; $\left.\mathrm{R}^{2}=0.9041\right)$; body weight (Bw) and maximum body height $(\mathrm{H})$ : group $\mathrm{C} \quad(\mathrm{r}=0.9900$; $\left.\mathrm{y}=0.0303 \mathrm{x}+2.1249 ; \mathrm{R}^{2}=0.9818\right)$, vs. group $\mathrm{E}$ $\left(\mathrm{r}=0.9530 ; \mathrm{y}=0.0134 \mathrm{x}+3.4178 ; \mathrm{R}^{2}=0.9095\right)$; body weight $(\mathrm{Bw})$ and body great perimeter $(\mathrm{P})$ : group C $\quad\left(r=0.9930 ; y=0.0887 x+5.7393 ; \quad R^{2}=0.9866\right)$, vs. group E ( $\mathrm{r}=0.9600 ; \mathrm{y}=0.0397 \mathrm{x}+9.0394$; $\left.\mathrm{R}^{2}=0.9220\right)$; body weight (Bw) and head length (Hl): group C ( $\mathrm{r}=0.9530 ; \mathrm{y}=0.0119 \mathrm{x}+2.5015$; $\left.\mathrm{R}^{2}=0.9097\right)$ vs. group $\mathrm{E} \quad(\mathrm{r}=0.9900$; $\left.\mathrm{y}=0.0092 \mathrm{x}+2.7158 ; \mathrm{R}^{2}=0.9820\right) ;$ body weight $(\mathrm{Bw})$ and caudal peduncle length $(\mathrm{CPl})$ : group
C $\left(\mathrm{r}=0.7750 ; \mathrm{y}=0.0084 \mathrm{x}+2.183 ; \mathrm{R}^{2}=0.6014\right)$, vs. group $E\left(r=0.980 ; y=0.0067 x+2.2208 ; R^{2}=0.9622\right)$.

Conclusion. The obtained values in this study, conducted in 2012, were confirmed by repeating the experiment in 2013, in the same conditions. In conclusion, we can say that based on data of the growth, development, changes in body form indices and growth density, can be estimated the future production of trout, with specific reference to the farming system used.

\section{REFERENCES}

1. Howland H.C., Merola S., Basarab J.R. (2004). The allometry and scaling of the size of vertebrate eyes. Vision Research, 44(11): 2043-2065.

2. Post J.R., Parkinson E.A. (2001). Energy allocation strategy in young fish: allometry and survival. Ecology, 82(4): 10401051.

3. Randall R.G., (2002). Using allometry with fish size to estimate production to biomass $(\mathrm{P} / \mathrm{B})$ ratios of salmonid populations. Ecology of Freshwater Fish, 11: 196-202. 